\title{
Optimization of candidate proteins for serological screening of Chlamydia trachomatis infection
}

\author{
X.B. Gao, M. Xiao, J. Wang, Y.J. Liu, Q.Z. Liu and M.L. Qi \\ Dermatology Department, Tianjin Medical University General Hospital, \\ Tianjin, China \\ Corresponding author: M.L. Qi \\ E-mail: qimanli360@163.com
}

Genet. Mol. Res. 14 (4): 12240-12246 (2015)

Received February 5, 2015

Accepted June 15, 2015

Published October 9, 2015

DOI http://dx.doi.org/10.4238/2015.October.9.12

\begin{abstract}
The aim of this study was to optimize candidate antigen proteins for serological screening of Chlamydia trachomatis infection. C. trachomatis positive serum and swabs of genital secretions were collected from 50 patients in the Tianjin Medical University General Hospital, as well as from 30 patients negative for C. trachomatis. Samples were assessed by colloidal gold assay in a sexually transmitted disease clinic as follows: serum antibodies for eight kinds of $C$. trachomatis immunodominant proteins (Pgp3, CPAF, CT143, CT101, CT694, CT875, CT813, and IncA) were detected, and two traditional gold standards, immunofluorescence and $C$. trachomatis cell culture of genital secretions, were used for comparison in order to determine the antigen protein combinations with the highest sensitivity and specificity. Of the 50 samples that tested positive for $C$. trachomatis infection by colloidal gold assay, 44 tested positive by micro-immunofluorescence, whereas 6 tested negative. In contrast, 14 samples tested positive by cell culture, whereas 36 tested negative. Serological results of the immunodominant protein combination of Pgp3, CT694, and CT875
\end{abstract}


shared positive coincidence rates of 97.73 and $92.86 \%$ with $C$. trachomatis micro-immunofluorescence and cell culture, respectively. No antibodies of the three proteins were detected in the 30 C. trachomatis samples that tested negative by colloidal gold assay; these samples also tested negative in C. trachomatis genital secretion culture. Overall, the combination of the three immunodominant proteins Pgp3, CT694, and CT875 had good sensitivity and specificity for serological screening of C. trachomatis infection, and the process was simple and easy to apply.

Key words: Chlamydia trachomatis; Immunodominant protein; Serological screening

\section{INTRODUCTION}

Genitourinary infection caused by Chlamydia trachomatis (C.t) has become the most common sexually transmitted disease worldwide, and the pelvic inflammatory disease, ectopic pregnancy, tubal infertility, miscarriage, stillbirth, and other complications caused by the infection have attracted increasing levels of attention (Workowski and Berman, 2011). Because of the occult nature of the symptoms (Wang, 2007), C.t-infected individuals have become important sources of infection reservoirs, which is the main cause of C.t prevalence. Early screening has demonstrated that identification of C.t-infected individuals was very important for C.t disease control, but all current detection methods rely on collection of urogenital secretions as specimens, and men were often discouraged by the pain of the drawing process required for C.t detection. Serological testing is one of the primary methods used for large-scale screening, and is more readily accepted. In order to find suitable antigen proteins for serological screening of C.t infection, we collected 50 sets of serum and genital secretion swabs determined to be positive for C.t by colloidal gold assay, and selected the recently reported eight C.t immunodominant proteins (Pgp3, CPAF, CT143, CT101, CT694, CT875, CT813, and IncA) (Wang et al., 2010) for detection of corresponding serum antibodies. The results were compared with those of two traditional gold standards, serum micro-immunofluorescence (MIF) and C.t cell culture of genital secretions, to find the best combination of C.t immunodominant proteins for C.t detection, and to provide evidence for the efficacy and utility of serological screening of C.t infection.

\section{MATERIAL AND METHODS}

\section{Clinical sample selection}

C.t positive serum and genital secretion swabs were collected from 50 subjects in the STD clinic of the Tianjin Medical University General Hospital from March to December 2013; C.t infection was detected by colloidal gold assay (Lanzhou Ya Hua Biotechnology Co., Ltd., Lanzhou, China). This study was approved by the Ethics Committee of Tianjin Medical University, and patients provided informed consent. 


\section{Reagents}

Bovine serum albumin and Escherichia coli competent BL-21 cells were obtained from Beijing Dingguo Biotechnology Co., Ltd. (Beijing, China); horseradish peroxidaselabeled goat anti-human $\mathrm{IgG}$ and goat anti-mouse $\operatorname{IgG}$ were obtained from Beijing Aobosen Biological Technology Co., Ltd. (Beijing, China), along with FITC-labeled green fluorescent goat anti-mouse $\mathrm{IgG}$ and Cy3-labeled red fluorescent goat anti-human $\mathrm{IgG}$; single-component chromogenic reagent ABTS were purchased from Shanghai Shengtang Biotechnology Co., Ltd. (Shanghai, China); GTS Mangbeads were from GenScript Corporation (Piscataway, NJ, USA); glutathione-precoated ELISA96 plates were purchased from Pierce Biotechnology (Rockford, IL, USA); the recombinant plasmids for MIF of 10 kinds of protein and the pGEX6P-2 vector, purified EB, Pgp3, MOMP, and OmcBc mouse-derived monoclonal antibodies were provided by professor Zhong Guangming, the Department of Microbiology and Immunology, Antonio Health Science Center, San Antonio, TX, USA; recombinant plasmids were sequenced by Beijing Sino gene Co. Ltd. (Beijing, China).

\section{Test methods}

\section{Detection of C.t by ELISA}

Purification and serum antibody detection of C.t immunodominant proteins by ELISA were performed as previously described (Wang et al., 2010; Xiao et al., 2013).

\section{Detection of C.t by MIF}

MIF to detect serum anti-C.t-specific IgG antibodies was performed as previously described (Saadouni et al., 2013) with modifications as follows: To prepare the antigen pieces of the C.t elementary body (EB), the purified EB solution was diluted by phosphate buffered saline (PBS) at a ratio of 1:100, and was dripped on a circular coverslip with 10 $\mathrm{mL}$ per antigen spot after autoclaving; after natural drying, the sample was fixed with $1 \%$ paraformaldehyde. After natural drying, the first antibody was added: 1) MOMP was diluted with PBS, and $8 \mu \mathrm{L}$ taken to cover the C.t antigen spot, followed by incubation at $37^{\circ} \mathrm{C}$ for $1 \mathrm{~h}$ in a wet box. 2) Serum samples of patients infected with C.t were diluted with PBS, and $8 \mu \mathrm{L}$ diluted serum was used to re-cover the C.t antigen spots, which were incubated at $37^{\circ} \mathrm{C}$ for $1 \mathrm{~h}$ in the wet box.

Samples were subsequently washed with PBS 3 times and once with distilled water, and allowed to air dry. Secondary antibodies consisting of $10 \mu \mathrm{L}$ antibody dilutions of FITC-labeled green fluorescent goat anti-mouse $\lg \mathrm{G}$ and $\mathrm{Cy} 3$-labeled red fluorescent goat anti-human $\operatorname{IgG}$ were added, and the samples were again incubated at $37^{\circ} \mathrm{C}$ for $1 \mathrm{~h}$ in the wet box. After washing three times by PBS and distilled water, samples were allowed to dry naturally.

Anti-decay fluorescent sealing reagent was dropped onto a clean glass slide and dried in the dark; slides were saved at $4^{\circ} \mathrm{C}$ in a cartridge. A fluorescence oil microscope was used to observe the samples. Excluding non-specific fluorescent highlights, samples were judged as positive for C.t infection when the coincident highlights of green and red fluorescence exceeded 10 in each field. 


\section{Cell culture and polymerase chain reaction (PCR)}

Cell culture was performed as described by Fresse et al. (2010) and Zhong et al. (2014). PCR amplification was performed as described previously (Li et al., 2004; Cook et al., 2005; He et al., 2009).

\section{RESULTS}

After SDS-PAGE electrophoresis and Coomassie blue staining, GTS Mangbead purified Pgp3, GST-CPAF, GST-CT143, GST-CT101, GST-CT694, GST-CT875, GST-CT813, GST-MOMP, GST-IncA, GST-Hsp60, and GST proteins expressed by empty plasmid showed clear target bands. Results from testing the 50 samples are shown in Table 1.

\begin{tabular}{lccc} 
Table 1. Detection of serum antibodies of eight kinds of immunodominant proteins. \\
\hline Proteins & Positive (cases) & Negative (cases) & Positive rate (\%) \\
\hline Pgp3 & 44 & 6 & 88 \\
CPAF & 38 & 12 & 76 \\
CT143 & 37 & 13 & 74 \\
CT101 & 36 & 14 & 72 \\
CT694 & 33 & 17 & 66 \\
CT875 & 31 & 19 & 62 \\
CT813 & 30 & 20 & 40 \\
IncA & 24 & 26 & 48 \\
\hline
\end{tabular}

\section{MIF and culture of C.t.}

In the collected 50 serum samples, 44 were found to be positive for C.t (88\%) and 6 were negative (12\%) following MIF testing, as shown in Figure 1; of the 50 samples collected of genital secretions, results were positive for $14(28 \%)$ and negative for $36(72 \%)$ using C.t cell culture (Figure 2).

\section{Optimization of antigen protein combinations for serological screening}

Through the comparison of the serum antibody detection and MIF and C.t culture, the combination of Pgp3, CT694, and CT875 proteins was selected. The antibody positive rate of the three proteins shared positive coincidence rates of 97.73 and $92.86 \%$ with MIF and cell culture, respectively. No antibodies of the three proteins were detected in the 30 samples determined to be negative for C.t infection by colloidal gold assay; these samples also tested negative in C.t genital secretion culture. The antibody detection of the three proteins, C.t secretion culture, and C.t PCR of the 6 MIF negative serum samples (from the original 50 colloidal gold positive samples) are shown in Table 2. Cell culture of genital secretions is shown in Figure 3. 


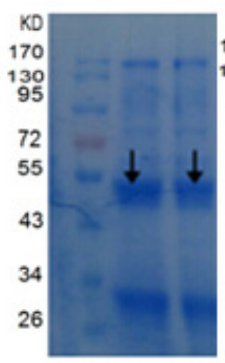

a

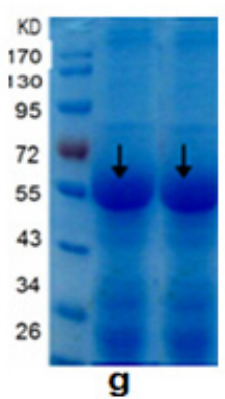

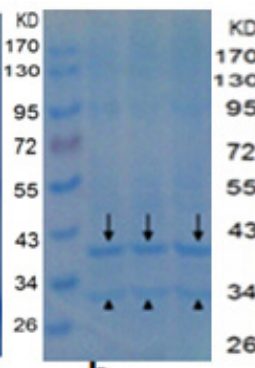

b

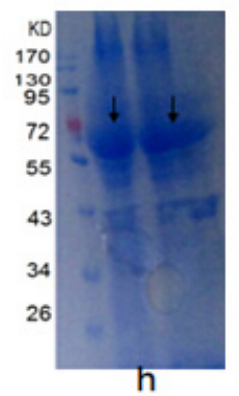

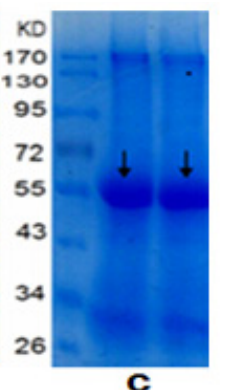

C

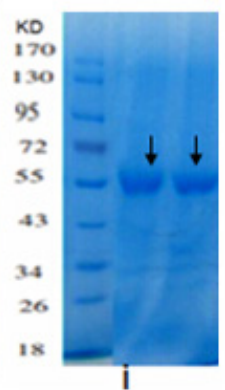

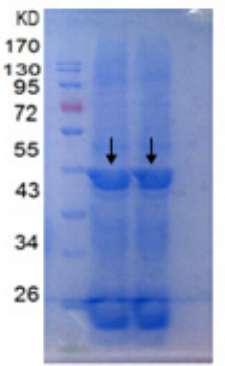

d

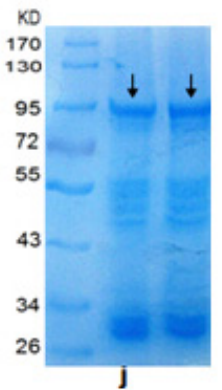

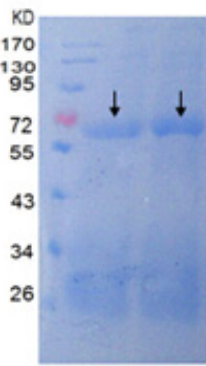

e
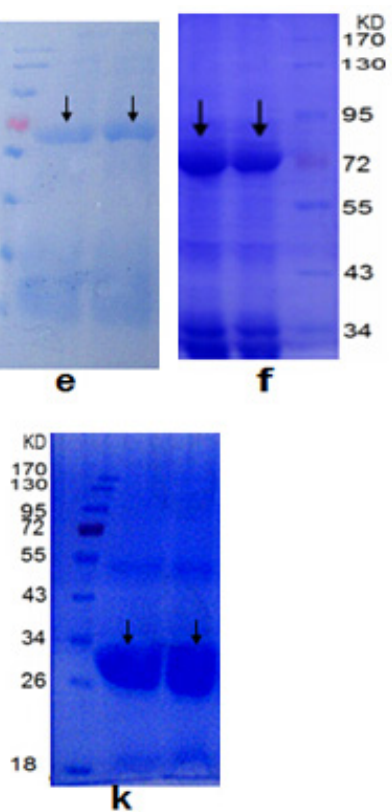

Figure 1. SDS-PAGE analysis of 11 kinds of purified Chlamydia trachomatis fusion proteins. a. GST-Pgp3 (relative molecular mass of $56 \mathrm{kDa}$ ); b. Two fragments after degradation of GST-CPAF (relative molecular masses of CPAFn and CPAFc were 29 and $35 \mathrm{kDa}$, respectively); c. GST-CT143 (relative molecular mass of $57 \mathrm{kDa}$ ); $\mathbf{d}$. GST-CT101 (relative molecular mass of $43 \mathrm{kDa}$ ); e. GST-CT694 (relative molecular mass of $60 \mathrm{kDa}$ ); f. GSTCT875 (relative molecular mass of $68 \mathrm{kDa}$ ); g. GST-CT813 (relative molecular mass of $55 \mathrm{kDa}$ ); $\mathbf{h}$. GST-MOMP (relative molecular mass of $68 \mathrm{kDa}$ ); i. GST-IncA (relative molecular mass of $55 \mathrm{kDa}$ ); j. GST-Hsp60 (relative molecular mass of $84 \mathrm{kDa}$ ); k. GST expressed by empty plasmid (relative molecular mass of $26 \mathrm{kDa}$ ).
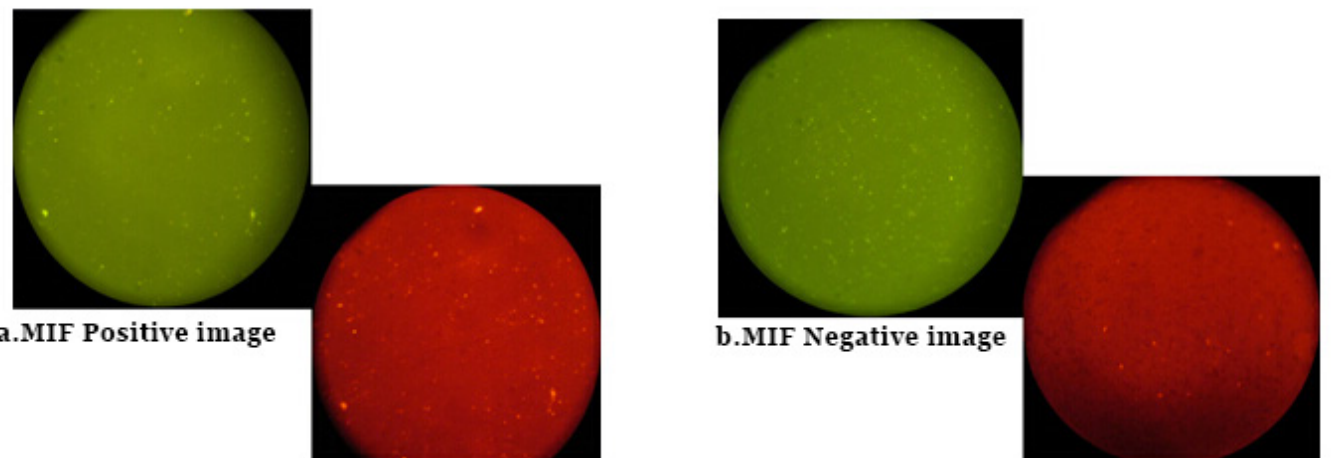

Figure 2. Immunohistochemical analysis of micro-immunofluorescence expression. Fluorescence highlights in the green vision field were Chlamydia trachomatis elementary body stained by FITC-labeled goat anti-mouse IgG, and the highlights in the red vision field were the deposition sites of serum anti-Ct-EB IgG antibodies stained by Cy3labeled goat anti-human IgG. Excluding non-specific fluorescent highlights, if the highlights in the green and red fields corresponded, they would be deemed as MIF positive images (a), and otherwise as negative (b). 
Table 2. Results of six micro-immunofluorescence-negative sera tested by other methods.

\begin{tabular}{|c|c|c|c|c|c|}
\hline Specimen No. & Pgp3 antibody & CT694 antibody & CT875 antibody & C.t culture of secretions & C.t plasmid amplification of secretions \\
\hline 1 & + & + & + & + & + \\
\hline 2 & + & + & + & - & + \\
\hline 3 & + & + & - & - & + \\
\hline 4 & + & + & - & - & + \\
\hline 5 & + & - & - & - & + \\
\hline 6 & + & - & - & - & + \\
\hline
\end{tabular}

C.t $=$ Chlamydia trachomatis.

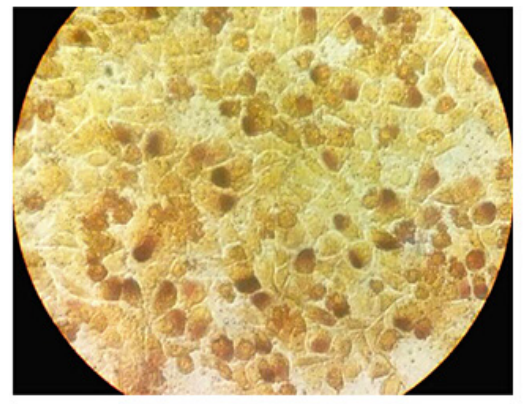

a. Cell culture positive image (Marked amplification multiples $\mathbb{Q}^{* *}$ )

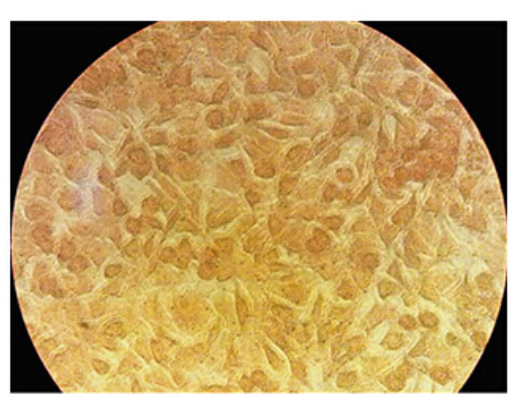

b. Cell culture negative images

(Marked amplification multiples ${ }^{* *}$ )

Figure 3. Cell culture of genital secretions (400X magnification). a. Image of positive cell culture; b. Image of

\section{DISCUSSION}

C.t screening plays an important role in the early detection and treatment of asymptomatic C.t infected individuals, as well as in the control of C.t spread and prevalence.

MIF is a serological method for C.t detection, considered the traditional gold standard for C.t serological diagnosis. It has high sensitivity and specificity; however, the method is cumbersome and antigen preparation is complex, requiring the expertise of experienced professionals. Furthermore, the judgment of results to a certain extent can be affected by subjective factors, and fluorescent antibodies are easily quenched; hence, the procedure is unsuitable for routine clinical performance and the detection of a large number of samples (Xue et al., 2009).

C.t cell culture is the gold standard for detection of the local presence of live C.t. However, the method has low positive rates and a complex operation, and is time-consuming and requires an expensive incubator and is therefore not suitable for routine usage and large-scale screening.

Serum antibody detection of C.t currently cannot be used to diagnose C.t infection in the clinic; however, the method has the advantages of low cost, ease of operation, and rapid procurement of results, which makes it a candidate for C.t infection screening. In order to find suitable antigen proteins for C.t infection screening and to improve the sensitivity and specificity of antibody screening, we selected eight C.t immunodominant proteins and compared the antibody test results with those of traditional MIF and cell culture methods. The results showed that the antigen combination of Pgp3, CT694, and CT875 shared consistent rates of 97.73 and $92.86 \%$ with the MIF and Chlamydia culture results. The three kinds antigen protein antibodies could also be detected in the 6 colloidal gold positive but MIF negative sera 
samples, and nucleic acid was positive for Chlamydia secretions, which confirmed that the six sera were indeed positive C.t samples. Therefore, this procedure had good sensitivity with the combination of the three antigen proteins to detect serum antibodies. An added benefit is that the three candidate antigen proteins are C.t specific (Fresse et al., 2010; Bullock et al., 2012; Chen et al., 2014) and have no cross-reaction with genitourinary bacteria, which can ensure the specificity of the antibody. No antibodies of the three proteins were detected in 30 samples from patients negative for C.t infection, as determined by colloidal gold assay; these samples also tested negative in C.t genital secretion culture.

We note, however, that screening results cannot fully represent clinical test results for C.t infection. Positive screening results only provide the possibility that the serum donors are likely infected by C.t, and the collection of genital secretions are required for testing by the MIF gold standard method or other currently available methods, in order to confirm the diagnosis.

\title{
Conflicts of interest
}

The authors declare no conflict of interest.

\section{ACKNOWLEDGMENTS}

\author{
Research supported by the National Natural Science Fund (\#81201355).
}

\section{REFERENCES}

Bullock HD, Hower S and Fields KA (2012). Domain analyses reveal that Chlamydia trachomatis CT694 protein belongs to the membrane-localized family of type III effector protein. J. Biol. Chem. 33: 28078-28086.

Chen YS, Bastidas RJ, Saka HA, Carpenter VK, et al. (2014). The Chlamydia trachomatis type III secretion chaperone $\mathrm{Slc} 1$ engages multiple early effectors, including TepP, a tyrosine-phosphorylated protein required for the recruitment of CrkI-II to nascent inclusions and innate immune signaling. PLoS Pathog. 10: e1003954.

Cook RL, Hutchison SL, Østergaard L, Braithwaite RS, et al. (2005). Systematic review: noninvasive testing for Chlamydia trachomatis and Neisseria gonorrhoeae. Ann. Intern. Med. 142: 914-925.

Fresse AS, Sueur JM and Hamdad F (2010). Diagnosis and follow-up of genital chlamydial infection by direct methods and by detection of serum IgG, IgA and secretory IgA. Indian J. Med. Microbiol. 28: 326-331.

He L, Wu YM, Li ZY, Zhou X, et al. (2009). Comparative study on three testing methods for Chlamydia trachomatis screening. Prog. Mod. Biomed. 9: 4273-4275.

Li ZY, Wu YM, Chen CQ and Yu MJ (2004). Expression and identification of major outer membrane protein of Chlamydia trachomatis in Escherichia coli. Chin. J. Dermatol. 37: 709-711.

Saadouni A, Tbai N and Takourt B (2013). Comparison of two techniques for serological diagnosis of chlamydial infections: MIF and immunoblotting. Ann. Biol. Clin. 71: 663-666.

Wang J, Zhang Y, Lu C, Lei L, et al. (2010). A genome-wide profiling of the humoral immune response to Chlamydia trachomatis infection reveals vaccine candidate antigens expressed in humans. J. Immunol. 185: 1670-1680.

Wang QQ (2007). Attaches great importance to the genital tract Chlamydia trachomatis infection prevention and control. Chin. J. Dermatol. 40: 257-259.

Workowski KA and Berman SM (2011). Center for Disease Control and Prevention Sexually transmitted diseases treatment guidelines. Clin. Infect. Dis. 53: S59-S63.

Xiao M, Wang J, Liu QZ and Qi ML (2013). Detection of antibodies against immunodominant proteins against Chlamydia trachomatis in the sera of patients with urogenital Chlamydia trachomatis infection. Chin. J. Dermatol. 46: 889-891.

Xue YH, Zheng HP, Xue XJ, XZ Wu, et al. (2009). Comparison of enzyme-linked immunosorbent assay and direct fluorescent antibody for the detection of Chlamydia trachomatis. Southern China J. Dermato-Venereology 16: 38-40.

Zhong MY, Yin YP and Wei WH (2014). Chlamydia trachomatis several realizations of somatic cells in primary culture. China AIDS Sexually Transmitted Dis. 1: 0066. 